\title{
Non-destructive discrimination of avocado fruit ripeness using laser Doppler vibrometry
}

\author{
Sandra Landahl and Leon A. Terry*
}

Plant Science Laboratory, Cranfield University, Bedfordshire, MK43 OAL, UK

*Corresponding author e-mail address: 1.a.terry@ cranfield.ac.uk

\begin{abstract}
Consumers increasingly desire ready-to-eat avocado fruit, yet if suppliers fall short of customer expectations, complaints follow incurring considerable cost and waste. In the avocado sector, wastage due to destructive testing and inaccurate assessment of firmness is significant. The aim of this study was to evaluate whether non-destructive laser Doppler vibrometry (LDV) was capable of assessing avocado ripeness. Data were sourced from two trials using preclimacteric imported 'Hass' avocado fruit originating from Chile and Spain, ripened at 12 and $18{ }^{\circ} \mathrm{C}$, respectively. Standard force-deformation measurements, and either single or simultaneous dual vibration time signals were recorded during shelf-life, and assessed against respiration and nonstructural carbohydrate content. Resonant frequencies measured of fruit by means of LDV decreased two- to four-fold during ripening and this corresponded with a concomitant decrease in firmness ( $253 \mathrm{~N}$ to $2 \mathrm{~N}$ ). The capability of the LDV system to non-destructively discriminate between ripeness stages was demonstrated.
\end{abstract}

Keywords: Persea americana, firmness, impulse response, quality control, mannoheptulose 


\section{Introduction}

The Organisation for Economic Co-operation and Development (OECD, 2004) recommends that quality of avocado fruit is rated according to size, estimated oil content, absence of defects and fruit firmness. However, it has been shown that avocados often do not soften uniformly due to physiological gradients (Landahl et al., 2009). The inherent heterogeneity within a fruit and between fruit in a consignment (Donetti and Terry, 2012) provides a challenge for distributors. At present up to $10-30 \%$ of avocado fruit are wasted due to destructive testing during grading and other packhouse activities and a further 5\% is lost at retail (Terry et al., 2011). The introduction of LDV systems to non-invasively assess avocado firmness, and indeed firmness of other fruit types where the industry is still reliant on destructive firmness testing as part of routine quality control, may help to reduce waste by as much as $10 \%$.

Consumers in the UK prefer ready-to-eat fruit and typically, eating firmness is achieved at 4.4 to $6.7 \mathrm{~N}$ according to Arpaia et al. (2015). Standard firmness evaluation by penetrometer (Magness-Taylor firmness) is inadequate in terms of accuracy, and its destructive nature. To avoid fruit waste due to destructive testing, many studies have reported on attempts to predict firmness by non-destructive techniques (Arzate-Vázquez et al., 2011, Magwaza and Tesfay, 2015). A number of non-destructive techniques have been developed to assess avocado quality in a broader sense. For instance, near infrared spectroscopy (NIRS) has been shown with variable success to predict maturity or oil content in avocado (Olarewaju et al. 2016, Ncama et al., 2018). Impulse response techniques (vibrometry) has been used to evaluate texture of several other commodities e. g. pear and grape, (Oveisi et al., 2014, Zhang et al., 2015, Trnka et al., 2016). In this study, non-destructive laser Doppler vibrometry (LDV) was investigated as possible technique to assess firmness in the packhouse before fruit are shipped to supermarkets. 
LDV has been used to evaluate fruit quality (Terasaki et al., 2001, Blahovec et al., 2008, Landahl and Terry, 2012, Zhang et al., 2015), and is based on the Doppler-effect which encompasses the frequency shift of back scattered light from a moving surface. Changing the optical path length per unit of time manifests itself as the Doppler frequency shift. Displacement measurement is better suited for low frequency measurements than velocity measurement and therefore should lend itself to evaluating fruit. LDV counts the bright-dark fringes on the detector. The resonant frequency (RF) of objects is measured when energy is applied to an object (e.g. a tap, or a sound sweep). RF is influenced by shape, mass and structure of the object, and in the case of fruit can relate to softness.

The aim of this study was to evaluate whether non-destructive LDV was capable of assessing cv. 'Hass' avocado firmness as model fruit. To demonstrate that laser vibrometry is capable of providing fast, robust and reliable information to implement industrial screening strategies for the assessment of firmness and damage detection in fruit and vegetables. To develop the excitation, detection and signal processing requirements for the measuring system. This will include assessment of the discrimination capabilities of the system, integrating appropriate physical measurements and statistical techniques to determine the level of sensitivity of the technology.

The objective of experiment 1 was to demonstrate on different sized fruit an impact device that is driven automatically, which provides a sharp impact to yield a signal easy to process into significant data. The objective of experiment 2 was to show that ripeness differentiation can be found by means of calculating fruit signals throughout ripening, in order to predict the readyto-eat stage measured by means of physiological and physical methods. 


\section{Materials and methods}

\subsection{Plant material and physical tests}

Imported avocado fruit cv. 'Hass' were supplied by M. W. Mack Ltd. (Kent, UK). Fruit were not treated with 1-methylcyclopropene. Fruit were supplied from Chile (experiment 1) size codes 18 (diameter mean $=68.55 \mathrm{~mm} \mathrm{SD}=1.815 \mathrm{~mm}$, mass day 0 mean $=237.7 \mathrm{~g} \mathrm{SD}=9.139 \mathrm{~g}$ ) and 22 (diameter mean=62.36mm SD=1.815mm, mass mean=187.2g SD=8.362g) and Spain size code 18 (mass range 203 to $243 \mathrm{~g}$ ). Fruit from Chile usually take 30 days to ship to the UK in containers with cool chain applied. Size 18 fruit were supplied by grower 826, Quillota province, Quillota, pack\# 34948, lot 143285 and size 22 fruit were from grower 627, Quillota province, La Cruz, pack\# 34958, lot 143289. Thirty avocados of each size were initiated to ripen at $20{ }^{\circ} \mathrm{C}$ for $24 \mathrm{~h}$ in their transport trays and then were kept at $12{ }^{\circ} \mathrm{C}$ in a $2 \times 3 \mathrm{~m}$ laboratory cool room in the dark for slow ripening. Repeated non-destructive LDV measurements were taken on days $0,1,3,5,7,9,11$ from all fruit resting in the space between two commerciallyused transport brushes (Fig. 1). A single point laser Doppler vibrometer was used to record signals repeatedly (CLV 700, Polytec UK Ltd., UK) from four impacts by a revolving wheel impact device. Impact and measurement were done around the equator of the fruit at approximately $90^{\circ}$ angle to each other. A self-fabricated little hammer consisting of a cable tie and a solid nylon ball (1 $\mathrm{cm}$ diameter) was attached to a motorized revolving wheel and used as the impact device (1.6 rpm at 24 V. Fig. 1). On day 3 and on the last day 12 fruit each were assessed destructively for firmness. Fruit flesh firmness was measured on two opposite sides of carefully knife-peeled fruit (Meyer and Terry, 2010). All texture tests were performed on a uni-axial testing machine (model 5542, Instron, MA) equipped with calibrated $500 \mathrm{~N}$ load cell. The machine was programmed (Bluehill 2, version 2.11, Instron) such that the probe indented the sample to a depth of $8 \mathrm{~mm}$ with the crosshead speed set at $20 \mathrm{~mm} / \mathrm{min}$. A flat cylinder probe of $8 \mathrm{~mm}$ diameter was used (Meyer and Terry, 2010). The mean value of two 
replicate measurements was calculated for each fruit. Firmness was expressed as the force required for tissue failure detected as bioyield $(\mathrm{N})$ in the force-deformation curve.

Spanish fruit from Malaga arrived in the UK within a week after picking (experiment 2). A cool chain was applied at $5-6{ }^{\circ} \mathrm{C}$ during lorry transport. On day 0 (baseline) fruit were randomised and labelled and allowed to warm up to ambient temperature. Treatment "box Ethyl": One hundred and forty avocados were transferred into gas tight boxes (ca. $325 \mathrm{~L}$ ) to be treated with ethylene for $24 \mathrm{~h}$ in a separate $18^{\circ} \mathrm{C}$ room. Controlled ripening was initiated using exogenous ethylene provided as a ready-made mix $(100 \mu \mathrm{mol} / \mathrm{mol}$ balanced with air; BOC Gases Ltd., Surrey, UK). Treatment "box air": One hundred and forty fruit were kept in boxes untreated and treatment "tray air": another one hundred and forty were kept untreated on open trays in a temperature controlled $18{ }^{\circ} \mathrm{C}$ room, so that controls within and outside gas tight boxes were produced in case the $24 \mathrm{~h}$ period inside a confined box raised respiration. All fruit were ripened on trays in the cool room at $18{ }^{\circ} \mathrm{C}$ for six days and during this time destructive and non-destructive tests were performed. Destructive assessment occurred on 8 fruit per treatment in sequence: firstly non-destructive LDV was recorded (details below), then firmness was measured destructively on two opposite sides of the fruit as previously described and last mesocarp tissue was snap frozen in liquid nitrogen for subsequent sugar analyses (thin slice cut across longitudinal axis of fruit). Destructive measurements were performed on days $0,2,3,4$ and $6(n=104)$ with only 8 fruit measured in total on day 0 , since no treatment was present at this time, therefore these fruit were labelled as "tray air". Sugar samples were only analysed from fruit tested on days $0,2,3$ and $6(\mathrm{n}=80)$. In addition, one hundred fruit per treatment were measured repeatedly by means of LDV on every test day $(n=300)$. Non-destructive measurements were carried out using two LDVs simultaneously, positioned along the longitudinal axis of the fruit: one LDV pointing closer to the stem end and one LDV pointing closer to the seed end of the fruit (CLV 700 and CLV 2534, Polytec UK Ltd., UK). Differently 
from experiment 1 , avocados were manually impacted with nylon-ball hammer and positioned on a moulded sponge. The fruit were impacted at three equidistant spots around the equator. Those three signal-pairs (10 ms each) were saved and processed.

\subsection{Physiological tests on Spanish avocados}

Two avocados each were held in four $3 \mathrm{~L}$ jars for $3 \mathrm{~h}$ on days $0,2,3,4$ (section 2.1 ), so the 8 fruit were paired into 4 replicates during this measurement. Gas samples were removed with repeated full withdrawal-injection displacements of a $50 \mathrm{~mL}$ plastic syringe (Meyer and Terry, 2010). Ethylene concentration was quantified using a gas chromatograph (GC 8340, Carlo Erba Instruments, Herts., UK) fitted with an EL 980 flame ionisation detector and DP800 integrator (Thermoquest, Herts., UK). Oven and detector temperatures were set at $100{ }^{\circ} \mathrm{C}$. The $2 \mathrm{~m}$ long stainless steel column was packed with Porapak (Jones Chromatography, Mid Glamorgan, UK). Carbon dioxide was quantified using the same GC system with hot wire detection. The hot wire detector was operated at $120^{\circ} \mathrm{C}$ and the oven at $50{ }^{\circ} \mathrm{C}$. The $\mathrm{GC}$ was calibrated with certified gases by BOC (British Oxygen Company, Surrey, UK).

Non-structural carbohydrates (NSC) were extracted and quantified as previously described (Landahl et al., 2009). In summary, the recovered residue powder (150 mg) was mixed with 3 $\mathrm{ml}$ of $62.5 \%(\mathrm{v} / \mathrm{v})$ aqueous methanol, placed in a water bath with shaker, and then left to cool. Then the samples were filtered through a syringe driven filter unit $(0.2 \mu \mathrm{m}$, Millipore Corp., MA), and the clear extract analyzed. Briefly, NSC content in the avocado extracts was determined using a high performance liquid chromatography (HPLC) system, where extracts were injected onto a Rezex RCM-Monosaccharide Ca+ (8\%) column, 300 x 7.8 mm size (00H0130-K0, Phenomenex, CA). Eluted NSCs were detected by an evaporative light scattering detector (ELSD 2420, Waters, MA) and mixed standards were used and presence of each NSC quantified by comparing sample peak areas to the standards. 


\subsection{Laser Doppler vibrometry data processing}

Vibration time signals were acquired using a simple algorithm written in Labview@ (v. 9.0, National Instruments Corporation, Austin, TX). In experiment 1, sampling frequency was 20 kHz. Resonant frequencies were calculated using Matlab@ software (7.12.0.635 R2011a, The MathWorks Inc., MA) as follows: An exponential window was applied, Fast Fourier Transform (FFT) performed, and a high pass filter applied. Then all four frequency spectra were multiplied to reduce influence of noise and reduce peaks that were only detected in one of the signals. The first peak was recorded as RF of the first spheroidal mode for all fruit (Kadowaki et al., 2012). Stiffness $S$ was approximated according to the formula for spheres. $m$ is mass.:

$S=R F^{2} * m^{2 / 3}$

Eq.1

The data in experiment 2 with Spanish fruit were acquired with a sampling frequency of 40 $\mathrm{kHz}$. The three signals per laser were multiplied and the RF of the first peak recorded. One signal was calculated for each laser, so that two results per fruit were obtained.

In order to evaluate damping data processing consisted of: a band pass filter was applied, then the absolute value of the time signal was calculated to obtain all vibration lobes in the positive domain. An exponential function was applied as a vibration signal-envelope to obtain a coefficient describing the attenuation of the vibration time signal. This corresponds to the power-law frequency dependent acoustic attenuation:

$P(x+\Delta x)=P(x) e^{-\alpha(\omega) \Delta x}, \alpha(\omega)=\alpha_{0} \omega^{\eta}$

$\omega$ is the angular frequency, $P$ the pressure, $\Delta x$ the wave propagation distance, $\alpha(\omega)$ the attenuation coefficient, $\alpha_{0}$ and frequency dependent exponent $\eta$ range from 0 to 2 .

The acoustic attenuation of a wide range of viscoelastic materials can be expressed as this law. The coefficient corresponding to $P(x)$ in equation 2 was forced to one, in order to analyse only one damping value regarding the attenuation. 
In order to obtain RF, an exponential window was applied, a FFT was performed on data with band pass filter applied and frequency spectra were smoothed with the 'mssgolay' function then peak detection was carried out. The first spheroidal mode, which was at least $40 \%$ of the highest amplitude, resonant frequency at the highest amplitude, damping and the standard squared error (SSE) of damping were saved to a file. Before statistical analysis, damping values with an SSE higher than 1 were rejected. Stiffness, which is a structural property and dependant on geometry / shape was not calculated and mass and shape of the fruit have been neglected when comparing RFs, since the whole batch was one size grade and assumed of similar shape. A previous test on one avocado, which was measured 32-times in normal position and back to front regarding the two laser positions, had shown it was best to use the first spheroidal mode for consistency of resonant frequencies collected from one fruit. In addition, it was shown that it did not matter which laser was pointed at the stem end or the seed end (Student's t-test 79.6 $\%$ for the first frequency).

\subsection{Statistical analyses}

Analysis of variance (ANOVA) was carried out using Genstat for Windows, version 15 (VSN International Ltd., Herts., UK). Factors were day of test and size grade (experiment 1), or treatment and LDV measurement position (experiment 2). F-test required to be below $5 \%$. For LDV data of the Spanish fruit were used as blocks, since paired values of the same sample existed. Outliers were removed according to software's outputs for destructively measured samples (residual analysis).

A partial least square regression (PLS) was calculated and with this model a prediction was performed on repeatly measured fruit by means of Unscrambler@ (X, version 10.5.46655.794, CAMO software, Norway). For the model, thirteen cross validation segments were chosen with 8 samples each, in order to recreate groups of days shelf life $(n=5)$ and treatments $(n=3)$. Factor 1 explained $95 \%$ of the variability and factor 2 explained $5 \%$. The parameters describing 
the goodness of fit between predicted and measured values within this model during calibration and validation are indicated in Table 1 . The two sets of RF values of the repeatedly measured fruit $(n=300)$ were used to perform a prediction of those fruit's firmness with the above described model as calibration model.

\section{Results and discussion}

\subsection{Physiological changes in the Spanish avocados}

As expected, the climacteric peak indicated by respiration (Fig. 2a) and ethylene production (Fig. 2b) was found early after removing the fruit from cool storage. This indicates the initial stages of ripening. Highest peaks were produced from fruit kept in a gas-tight box without ethylene treatment. Respiration was lowest for fruit held on a tray, these fruit were also firmer initially. Ethylene production was lower and its peak delayed in fruit which were treated with exogenous ethylene at the start of the experiment. Destructively measured fruit, which were held in a box and treated with ethylene exogenously, showed higher frequencies on days 3, 4 and 6 (Table 2b), which corresponded to the observation of a slightly delayed ethylene peak. It appears that the exogenous ethylene application slightly delayed the endogenous ethylene production. Ethylene removal has been shown to modulate internal ethylene response pathways and affect abscisic acid concentration (Meyer, Chope, \& Terry, 2017).

Sucrose showed no significant differences over storage time or between treatments (mean=8.5

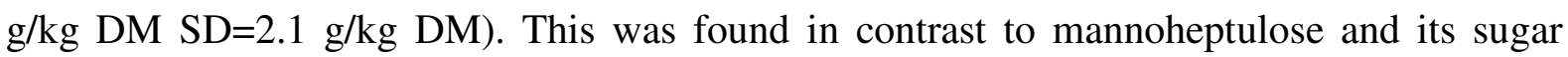
alcohol perseitol. Mannoheptulose (Fig. 2c) and perseitol content (on average from 20.2 to 6.8 g/kg DM 1.s.d.=2.4 g/kg DM) decreased significantly during ripening. Liu et al. (2002) suggested a role of mannoheptulose as a ripening inhibiting factor. It was suggested that past a certain threshold-value ripening occurs in avocado fruit. Therefore, it was expected that mannoheptulose concentration decreased while the fruit soften. Work by Donetti and Terry 
(2014) supported a role for mannoheptulose in the ripening process of avocados and pointed to a strong relation between concentration of this compound with fruit origin, which in turn might influence the consistency of fruit ripening within a batch.

\subsection{Analyses of LDV Spanish avocados}

It is common practise to destructively measure avocado fruit firmness to inform ripening stage and storage release. Fruit measured destructively showed that they were at eat-ripe stage on days 3 (Fig. 2d). Values of first spheroidal mode showed a wide range for predicting firmness of each fruit (Fig. 3a and b). When the fruit were soft (from day 4 onwards) the detection of the first resonant frequency became more difficult, yet these fruit would usually already have been sold at retail and thus not be sorted at the packhouse. The disconnection of the avocado skin as previously discussed by Landahl and Terry (2010) could account for the difficulty in measuring RF. In addition, the specific challenge with cv. 'Hass' avocado compared to other fruit is the often irregular surface of the skin, which could scatter a percentage of laser light, so it might not be detected.

All fruit that were measured showed a significant decrease in RF during ripening (Fig. 3a and b). According to the prediction values (Fig. 4) ready-to-eat stage would have been achieved on the same days as the destructively measured fruit from the same batch (Table2b).

A significant difference was found on day 3 between stem-end and seed-end of the 300 repeatedly measured avocados and a similar trend was found for the 24 fruit also measured destructively, where the values at the seed-end were $85 \mathrm{~Hz}$ higher. Ripened avocado flesh (mesocarp tissue) is visco-elastic, in contrast to the seed, which is rigid and elastic only, this influences the resonant frequency at this position. Otherwise, this would be in contradiction with previously found results, which found apical mesocarp tissue was firmer than distal tissue near the seed (Landahl et al., 2009). This was thought to be due to the suggested role of mannoheptulose as a ripening inhibitor (Liu et al., 2002) or the possible involvement of the 
seed in ethylene evolution and responsiveness to ethylene during ripening (Hershkovitz et al., 2008).

On days 0 and 6, damping values measured repeatedly at the stem-end of the Spanish fruit were higher than at the seed-end (Fig. 3c). As mentioned above the seed is firmer and more elastic than the fruit flesh. Significant differences between treatments were found for the 24 fruit later destructively measured, where at first the avocados held in boxes under air showed the highest values (Table 2b), but damping did not reveal a clear course of change. Damping values are used to describe the visco-elastic properties of the avocados. Eat-ripe avocado tissue has a creamy texture, which should correspond to higher attenuation. Only a small trend for higher values around day 3 show the potential predictive value of damping, but this test needs improvement.

LDV is dependent on structure of the measured object and this makes it a useful tool to assess texture of the fruit regardless of the origin, harvest practice or storage treatment of a batch; it has been related to cell wall polysaccharide changes in the past (Terasaki, et al., 2001). Research at cell membrane level to understand the mechanisms to reduce avocado batch heterogeneity have been explored to synchronise fruit ripening (Hernández et al., 2015, Defilippi et al., 2018).

\subsection{Comparison of destructive and non-destructively measured values and prediction}

Studies on consumer preferences have shown the importance of evaluating different quality aspects, e.g. dry matter content and firmness $(6.5 \mathrm{~N})$, in relation to discouraging factors, e.g. bruises or high price (Harker et al., 2019). The study described herein, focused on predicting firmness non-destructively to avoid wastage during grading. It is well accepted that benchmarking before shipping the fruit to retail markets is good commercial practice and improves acceptability of the final commodity. 
The average mass of the Chilean avocados in experiment 1 decreased linearly during slow ripening as expected due to water loss and respiration (day 0 (18) mean=237.7g SD=9.139g, day 5 (18) mean=228.4g SD=9.062g, day 11 (18) mean=219.6g SD=9.128g, day 0 (22) mean=187.2g SD=8.362g, day 5 (22) mean=179.3g SD=8.126g, day 11 (22) mean=171.5g $\mathrm{SD}=8.106 \mathrm{~g}$, respectively). In this experiment 1 , destructive measurements were only taken on two occasions and the firmness decreased significantly (Table 2a). The calculated stiffness values showed an approximately exponential decrease, even though only three measurement points were taken (Table 2a). This is a similar course as the exponential decrease in bioyield shown in experiment 2 (Fig. 3a). This shows the automated impact device was successful in providing meaningful signals that could be interpreted as expected. Experiment 1 was designed with a smaller amount of fruit to inform points of focus for experiment 2 , where two LDV were used, more destructively obtained data were collected and a large amount of fruit were measured repeatedly.

Avocados are climacteric fruit and usually a rapid decrease in firmness is initiated at the beginning of the respiration and ethylene production peaks (Arpaia et al., 2015). It has been shown by other authors that postharvest water loss has no effect on mechanical properties, but on the non-destructively measured impact responses (Harker et al., 2019). However, in the trial on Spanish fruit this could not be the major influencing factor, since the percentage of water content in the avocados showed no clear trend (Table $2 b$ ). The resonant frequency of the Spanish fruit decreased steeper until day 4 and decreased gentler thereafter (Fig. 3a and b). Usually, firmness of avocados decreases rapidly under shelf life conditions (Meyer and Terry, 2010) as seen herein, therefore a linear correlation between the resonant frequency and firmness is not expected. A model was created by PLS, which compared 104 avocados from the same batch measured non-destructively by LDV and destructively by means of uni-axial test. PLS is routinely used to interpret spectroscopy data, and in this study it was used to 
evaluate the ability of the non-destructively measured variables to predict ripening (Magwaza et al., 2014): The load at break of 300 repeatedly measured fruit was predicted with the calibration model. The result indicated a credible firmness development (Fig. 4). The external prediction had $90.7 \%$ variability explained in factor 1 , where the stem end RF contributed mainly to the model and seed end RF contributed mainly to the residuals. It has been shown previously that LDV measurements outperform other impact based techniques to evaluate firmness (Landahl and Terry, 2012). However, it remains to be investigated how robust a calibration is needed for good predictive performance over several seasons. This study concentrated on one batch as described, but used easily obtainable input values, i.e. RF and damping. Variability due to season and orchards is inevitable (Arpaia et al., 2015), therefore LDV is an effective tool to predict ripening at the grading step.

The study herein demonstrated that LDV is capable of differentiating between fruit of different firmness values and suggest RF threshold values for ready-to-eat fruit (Fig. 4). It is necessary to choose fruit of similar shape and mass (or weigh them to calculate the stiffness factor), in order to predict firmness of the fruit. Due to the heterogeneous ripening of mesocarp tissue within a fruit (Landahl et al. 2009) it was examined if the measurement of RF at opposite ends of the avocado fruits would give a differential value that could indicate fruit ripeness (Fig. 3a). This hypothesis was later abandoned, since the difference showed no clear trend and the RF values were in contradiction with former measurements of tissue stress-relaxation (Landahl et al. 2009), so that the RF values at the seed end of the fruit were interpreted as a result from mesocarp tissue and seed in combination (Landahl and Terry, 2012).

Avocado ripening has a dramatic effect on fruit rheological properties (Ortiz-Viedma, 2018). It is common practice to study these with quasi-static methods on a rheometer or uni-axial testing machine. LDV delivers rheological data in a fast and non-destructive way and can 
measure elasticity dynamically. The results herein confirmed the ability of LDV to describe temporal and spatial visco-elastic changes within fruit.

\section{Consclusion}

In conclusion, this study has shown that LDV is capable to non-destructively discriminate between ripeness stages of avocados and since this fruit is particularly challenging it is likely to be capable to be utilised on other fruit types as well. This study has not focussed on the comparison of fruit origin, size or treatment, but it has been shown that exogenous ethylene application after cold storage did not accelerate ripening as measured by firmness. A simple automated impact device for tapping the fruit in order to provoke vibration was developed and tested during this study. This will need further refinement to make it suitable for an industrial sorting line.

Several common inventory practices exist to hold and ripen avocado fruit until needed for retail. All these practices could be enhanced if the ripening stage of fruit could be nondestructively examined. In the current study, RFs measured non-destructively using LDV decreased in parallel with the firmness and physiological changes within avocado fruit. Thus, LDV could be applied to avocado and indeed other fresh produce types where firmness or structural integrity is vital in defining shelf life and consumer acceptability. Implementation on a commercial packing line and a robust calibration method will require further investigation. Typically a traffic light system would be envisaged, where fruit are sorted as 'right for marketing', 'not ready yet' / 'need to check again', or 'discard'. For a system like this, a large database has to be established or frequent calibration has to be performed to define thresholds in line with the commercial requirements. 


\section{ACKNOWLEDGEMENTS}

This work formed part of a larger FoodLink project (AFM 235) and was financially supported by the UK Government (Department for Environment, Food and Rural Affairs) and UK industry representatives (Lambda Photometrics Ltd, M. W. Mack Ltd., Marks and Spencer Plc, Unilever R\&D). The support from Prof. George Jeronimidis, University of Reading, is gratefully acknowledged. Data underlying this study can be accessed through the Cranfield University repository at https://doi.org/10.17862/cranfield.rd.11764188.v2.

\section{REFERENCES}

Arpaia, M.L., Collin, S., Sievert, J., Obenland, D. 2015. Influence of cold storage prior to and after ripening on quality factors and sensory attributes of 'Hass' avocados. Postharvest Biology and Technology 110:149-157.

Arzate-Vázquez, I., Jorge Chanona-Pérez, J., de Jesus Perea-Flores, M., Calderón-Domínguez, G., Moreno-Armendáriz, M. A., Calvo, H., Godoy-Calderón, S., Quevedo, R. and Gutiérrez-López, G. 2011. Image processing applied to classification of avocado variety Hass (Persea americana Mill.) during the ripening process. Food Bioprocess Technology 4:1307-1313.

Blahovec, J., Kuroki, S. and Sakurai, N. 2008. Precise correction in laser Doppler forced vibrology of soft products. Biosystems Engineering 99:156 - 160 .

Defilippi, B. G., Ejsmentewicz, T., Paz Covarrubias, M., Gudenschwager, O., Campos-Vargas, R. 2018. Changes in cell wall pectins and their relation to postharvest mesocarp softening of "Hass" avocados (Persea americana Mill.). Plant Physiology and Biochemistry $128: 142-151$.

Donetti, M. and Terry, L.A. 2012. Evaluation of factors affecting shelf-life and quality biomarkers of imported avocado fruit. Acta Horticulturae 934: 677-682. 
Donetti, M. and Terry, L.A. 2014. Biochemical markers defining growing area and ripening state of imported avocado fruit cv. Hass. Journal of Food Composition and Analysis 34:90-98.

Harker, F.R., Feng, J., Johnston, J.W., Gamble, J., Alavi, M., Hall, M., Chheang, S.L. 2019. Influence of postharvest water loss on apple quality: The use of a sensory panel to verify destructive and non-destructive instrumental measurements of texture. Postharvest Biology and Technology 148:32-37.

Hernández, I., Fuentealba, C., Olaeta, J. A., Lurie, S., Defilippi, B. G., Campos-Vargas, R., and Pedreschi, R. 2015. Factors associated with postharvest ripening heterogeneity of 'Hass' avocados (Persea americana Mill). Fruits 71(5):259-268.

Hershkovitz, V., Friedman, H., Goldschmidt, E. E., Ben-Arie, R., Feygenberg, O., Pesis, E. 2008. The role of ethylene response-pathway components in avocado ripening. Bornimer Agrartechnische Berichte 64:58.

Kadowaki, M., Nagashima, S., Akimoto, H., Sakurai, N. 2012. Detection of core rot symptom of Japanese pear (Pyrus pyrifolia cv. Kosui) by a non-destructive resonant method. Journal of the Japanese Society for Horticultural Science 81(4):327-331.

Landahl, S., Meyer, M. D., and Terry, L. A. 2009. Spatial and temporal analysis of textural and biochemical changes of imported avocado cv. Hass during fruit ripening. Journal of Agricultural and Food Chemistry 57(15): 7039-7047.

Landahl, S. and Terry, L. A. 2010. Textural, biochemical and micro-structural changes in mesocarp tissue of imported avocado from Peru during ripening. Acta Horticulturae 877:1063-1070.

Landahl, S. and Terry, L. A. 2012. Avocado Firmness Monitoring with Values Obtained by Means of Laser Doppler Vibrometry. IV International Conference Postharvest Unlimited 2011. Acta Horticulturae 945:239-245. 
Liu, X., Sievert, J., Arpaia, M. L., Madore, M. A. 2002. Postulated physiological roles of the seven-carbon sugars, mannoheptulose, and perseitol in avocado. Journal of the American Society for Horticultural Science 127:108-114.

Magwaza, L.S., Opara, U.L., Cronje, P.J.R., Landahl, S., Nieuwoudt, H.H., Mouazen, A.M., Nicolaï, B.M., Terry, L.A. 2014. Assessment of rind quality of 'Nules Clementine' mandarin fruit during postharvest storage: 2. Robust Vis/NIRS PLS models for prediction of physico-chemical attributes. Scientia Horticulturae 165:421-432.

Magwaza, L.S., Tesfay, S.Z. 2015. A Review of Destructive and Non-destructive Methods for Determining Avocado Fruit Maturity. Food Bioprocess Technology 8:1995-2011.

Meyer, M. D., and Terry, L. A. 2010. Fatty acid and sugar composition of avocado, cv. Hass, in response to treatment with an ethylene scavenger or 1-methylcyclopropene to extend storage life. Food Chemistry 121:1203-1210.

Meyer, M. D., Chope, G. A., and Terry, L. A. 2017. Investigation into the role of endogenous abscisic acid during ripening of imported avocado cv. Hass. JSci Food Agric 97:36563664.

Ncama, K., Magwaza, L. S., Poblete-Echeverrõ, C. A., Nieuwoudt, H., Tesfay, S. Z., Mditshwa, A. 2018. On-tree indexing of 'Hass' avocado fruit by non-destructive assessment of pulp dry matter and oil content. Biosystems engineering 174:41-49.

OECD. 2004. Avocados. International Standardisation of Fruit and Vegetables. 8-31.

Olarewaju, O. O., Bertling, I., Magwaza, L. S. 2016. Non-destructive evaluation of avocado fruit maturity using near infrared spectroscopy and PLS regression models. Scientia Horticulturae 199:229-236.

Ortiz-Viedma, J., Rodriguez, A., Vega, C., Osorio, F., Defilippi, B., Ferreira, R., Saavedra, J. 2018. Textural, flow and viscoelastic properties of Hass avocado (Persea americana 
Mill.) during ripening under refrigeration conditions. Journal of Food Engineering 219:62-70.

Oveisi, Z., Minaei, S., Rafiee, S., Eyvani, A., Borghei, A. 2014. Application of vibration response technique for the firmness evaluation of pear fruit during storage. J. Food Sci. Technol. 51(11):3261-3268.

Terasaki, S., Sakurai, N., Yamamoto, R., Wada, N., Nevins, D. 2001. Changes in cell wall polysaccharides of kiwifruit and visco-elastic properties detected by a laser Doppler method. J. Japan. Soc. Hort. Sci. 70(5):572-580.

Terry, L. A., Mena, C., Williams, A., Jenney, N. and Whitehead, P. 2011. WRAP (Waste \& resources action programme). Fruit and vegetable resource maps (RSC-008)

Trnka, J., Pavloušek, P., Nedomova, Š., Buchar, J. 2016. Time and frequency domain response of grape berries to non-destructive impact during the harvesting period. Journal of Texture Studies 47:24-33.

Zhang, W., Cui, D., Ying, Y. 2015. Nondestructive Measurement of Texture of Three Pear varieties and Variety Discrimination by the Laser Doppler Vibrometer Method. Food Bioprocess Technol. 8:1974-1981.

\section{FIGURES}

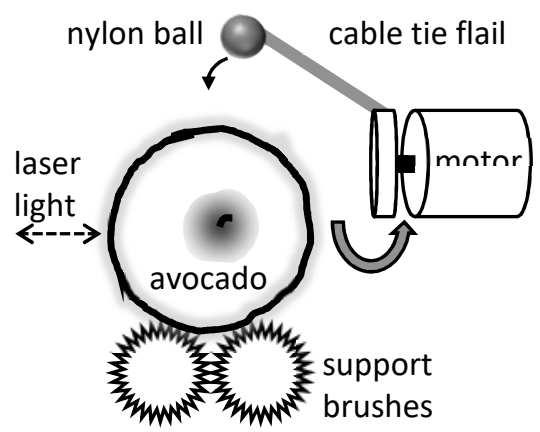

Fig. 1 Experiment 1, schematic of device to impact the Chilean avocados to perform RF measurement. 

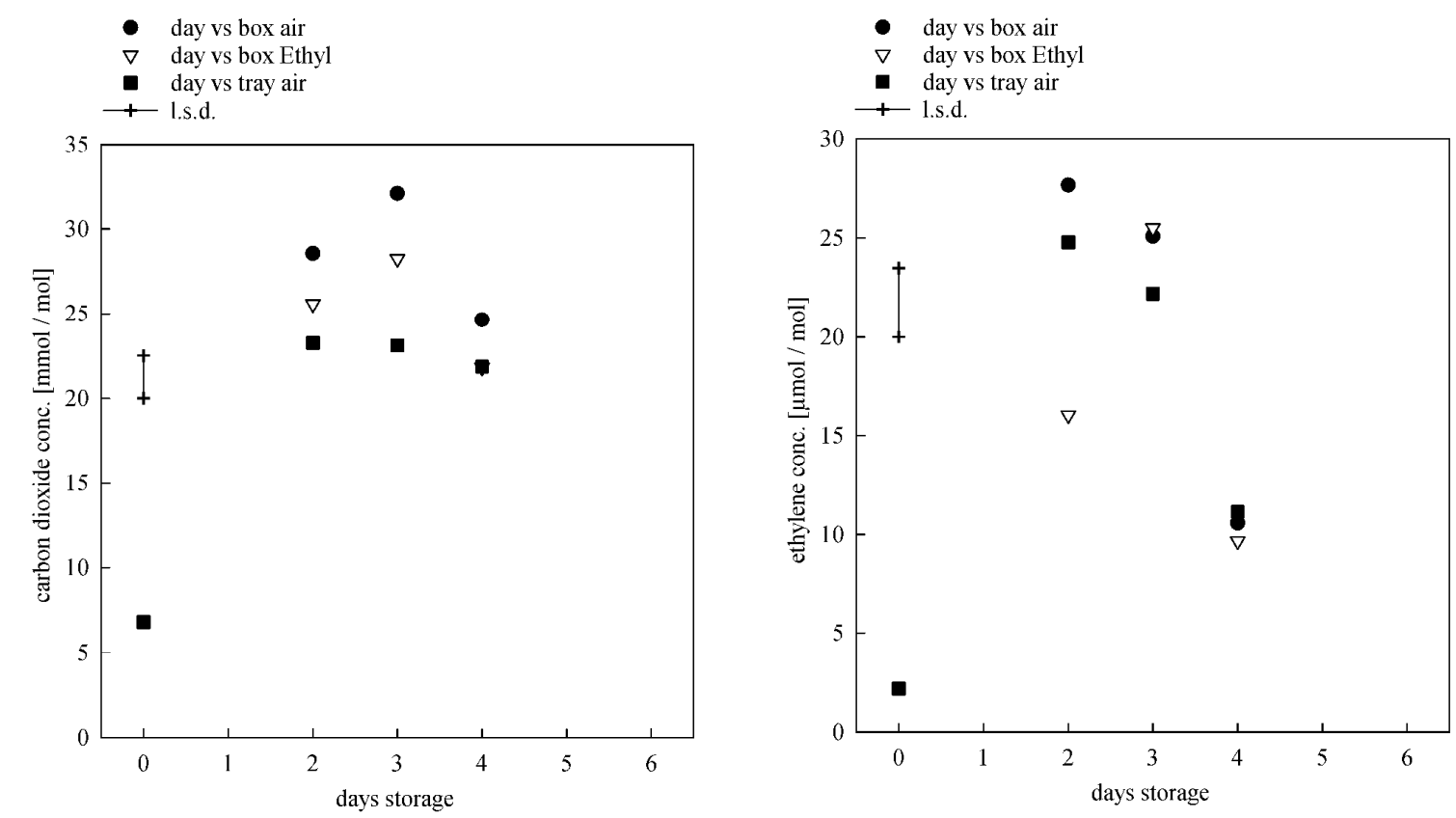

C

D
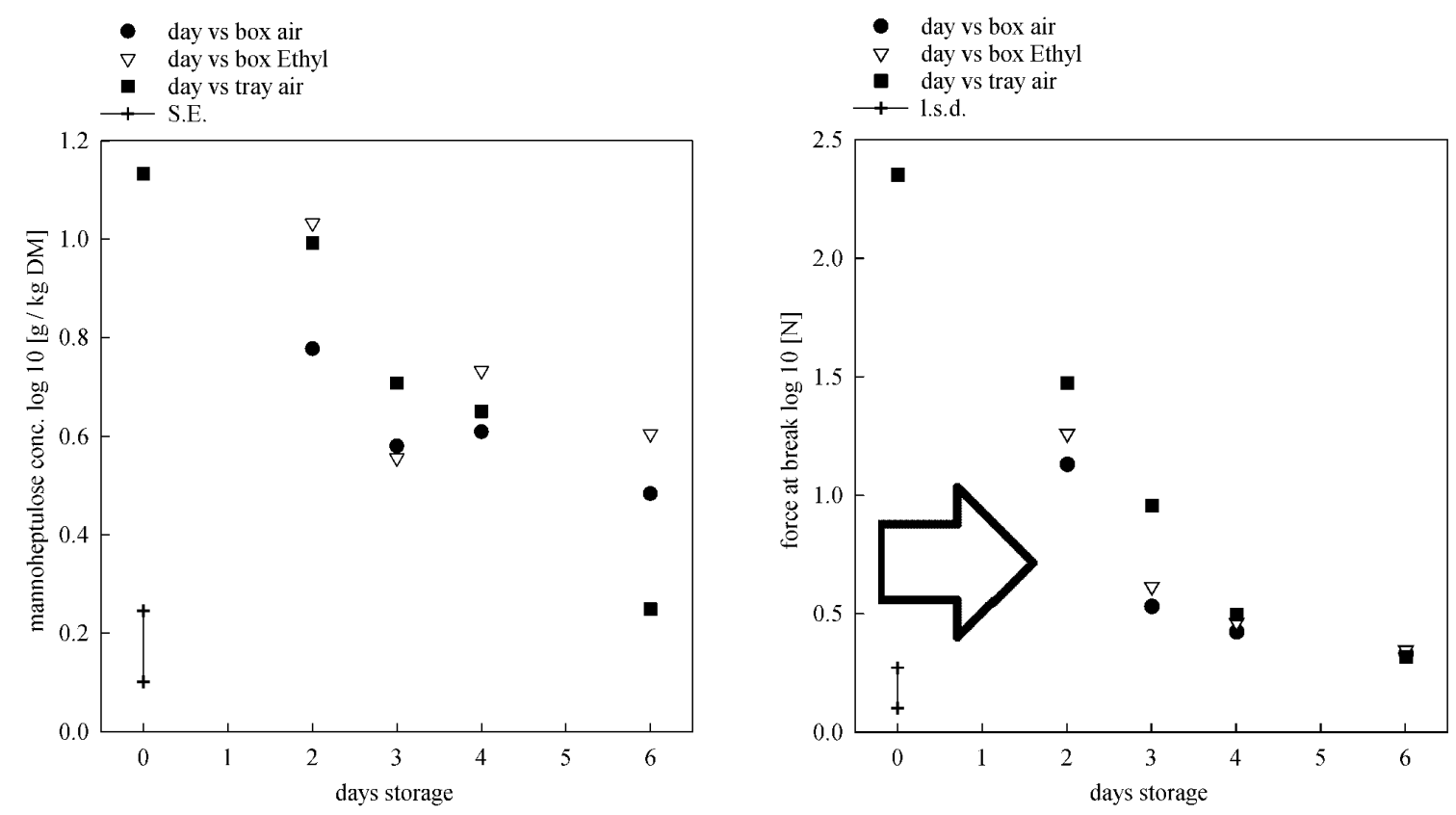

Fig. 2 Experiment 2 a) Indicator for $\mathrm{CO}_{2}$ respiration measured after incubating two size code 18 avocados per jar ( 8 fruit per point $=4$ measurements per point). Error bar indicates 1.s.d. $\mathrm{p}<0.05$. b) Indicator for ethylene production measured after incubating two size code 18 avocados per jar ( 8 fruit per point $=4$ measurements per point $)$. Error bar indicates 1.s.d. $\mathrm{p}<0.05$. 
c) Log.10 mannoheptulose concentration measured of avocados from Spain (8 each point).

Standard error bar $\mathrm{p}=0.074$. d) Log. 10 force at break of destructively measured avocados from Spain (8 each point). $\left.10^{0.826}\right)$. Error bar indicates 1.s.d. $\mathrm{p}<0.05$.

A

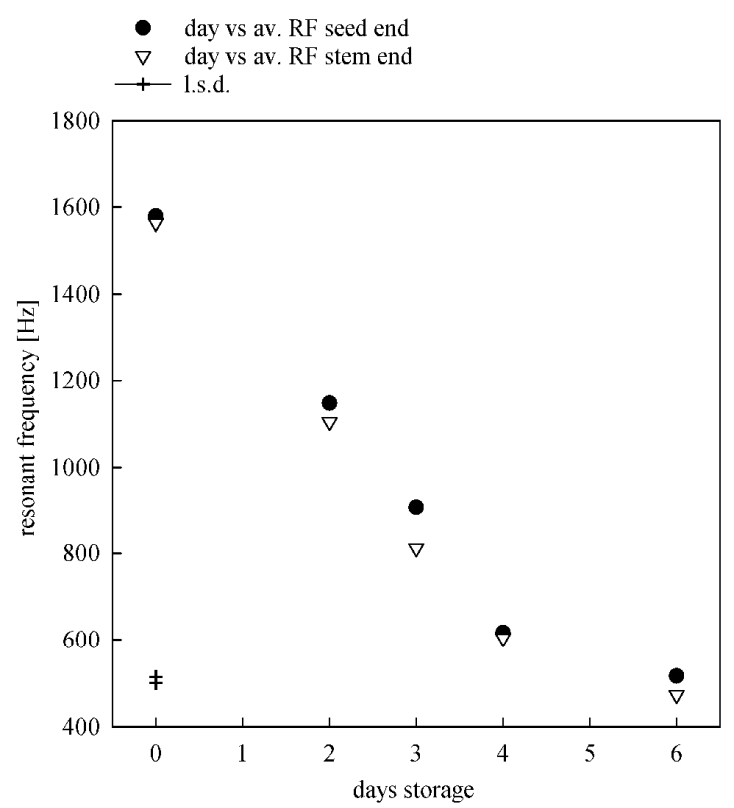

B

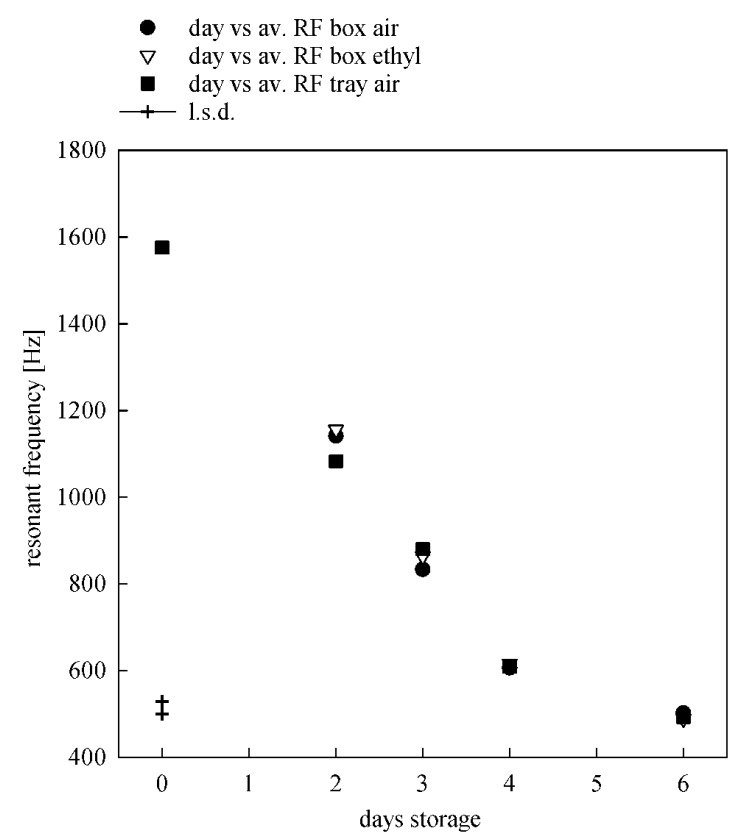


C

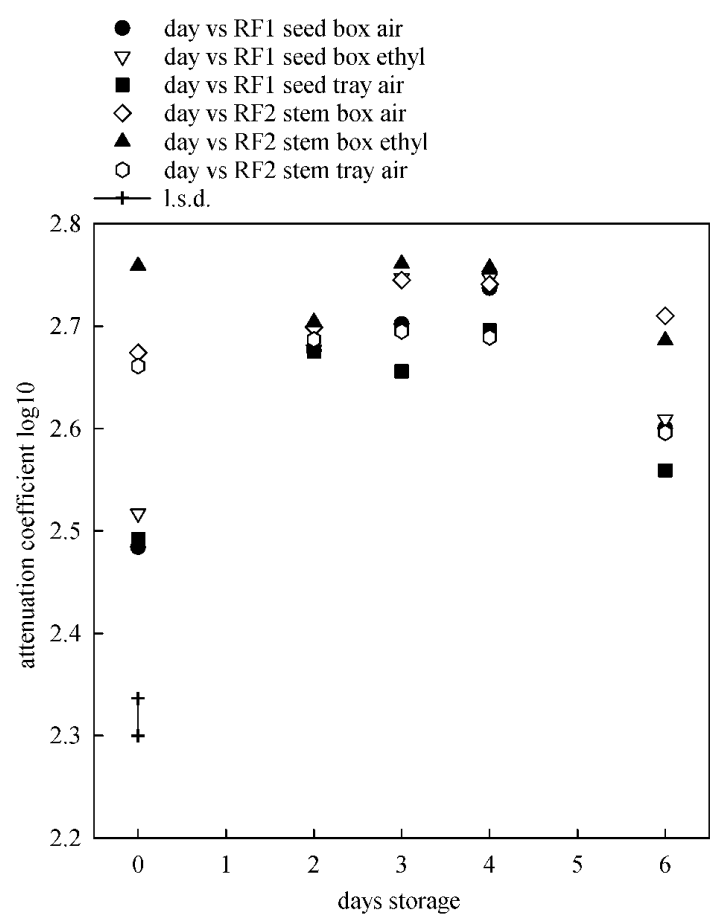

Fig. 3 Experiment 2 a) Average RF values of repeatedly measured avocados from Spain (100 each point). Main effect of stem end and seed end of fruit indicated separately. b) Main effect of treatment as grouped values. c) Log10 damping / attenuation values and error bar indicates 1.s.d. $\mathrm{p}<0.05$.

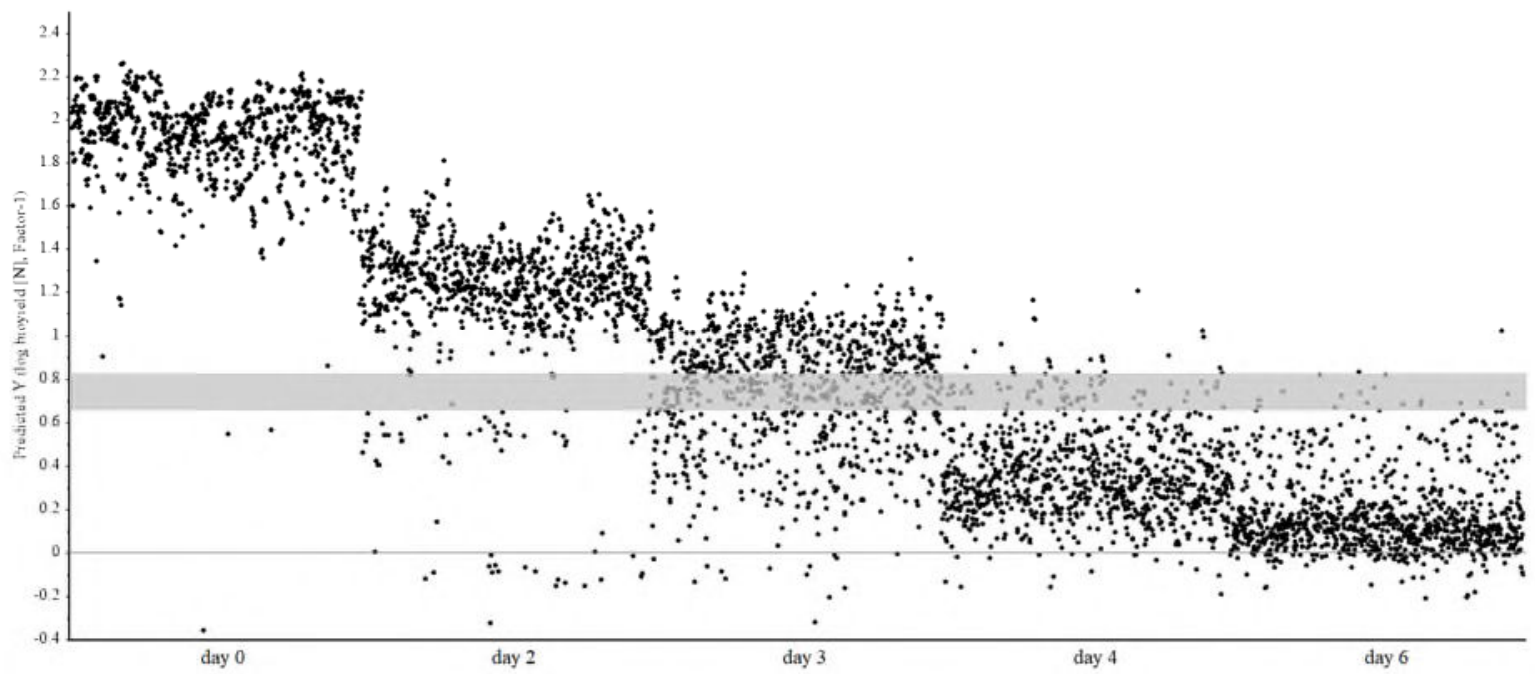

Fig. 4 Plot of predicted $\log 10$ force at break values related to 300 avocados repeatedly measured by means of LDV. Prediction achieved with PLS model from separately measured 
fruit from same batch (see section 2). Shelf-life days indicated. Grey box indicates ready-toeat range.

Table 1 PLS calibration model goodness of fit relating to avocados $(n=104)$ measured by means of LDV and uni-axial testing machine. In this case, two RF from first peak (seed and stem end) were used to create a model to predict $\log 10$ force at break / firmness values.

\begin{tabular}{lccccc} 
& Slope & Offset & R-square & RMSE & Bias \\
\hline Calibration & 0.78 & 0.19 & 0.78 & 0.30 & 0 \\
Validation & 0.68 & 0.24 & 0.72 & 0.36 & -0.028
\end{tabular}


Table 2 Mean values of avocado measurements during two different shelf-life trials as described above (exp. $112^{\circ} \mathrm{C}$ and exp. $\left.218{ }^{\circ} \mathrm{C}\right) .1 . s . d .=$ least significant difference $\mathrm{p}<0.05$. ${ }^{*}$ same fruit as repeatedly measured.

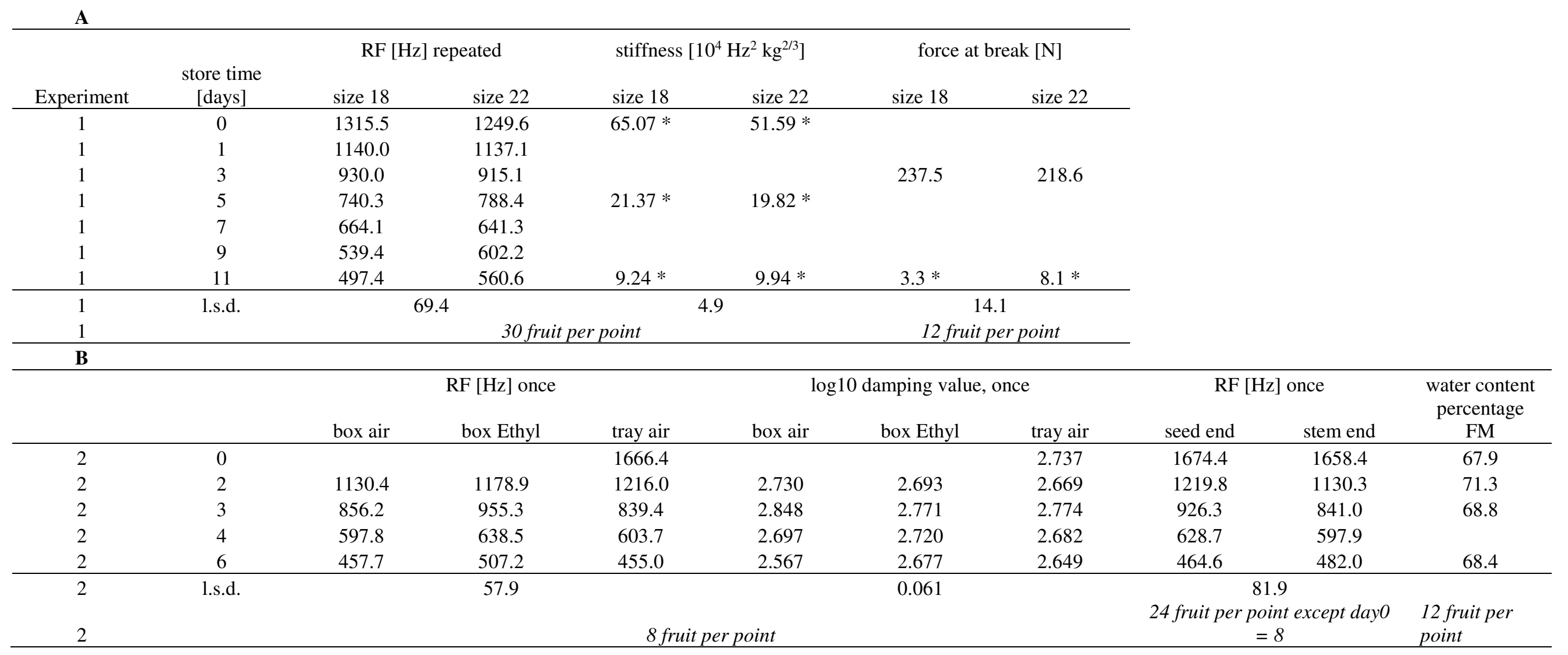

